Microbes Environ. Vol. 24, No. 3, 280, 2009

http://wwwsoc.nii.ac.jp/jsme2/ doi:10.1264/jsme2.ME07203e

\title{
Erratum
}

\section{Response to Environmental Stress as a Global Phenomenon in Biology: The Example of Microorganisms}

Bouachanh Thammavongs, Emmanuel Denou, Ghalia Missous, Micheline Guéguen, and Jean-Michel PanofF Food Microbiology Laboratory, IBFA-ISBIO, University of Caen Basse-Normandie, Esplanade de la Paix, 14032 Caen cedex, France

Volume 23, no. 1, p. 20-23, 2008. Page 21, the Table 1 is replaced by the table shown below.

Table 1. Non-exhaustive list of abiotic stresses

\begin{tabular}{|c|c|c|c|c|}
\hline Stress & Synonym, similar or close to & $\begin{array}{l}\text { Example of concerned } \\
\text { microorganisms }\end{array}$ & Taxonomic position & Reference \\
\hline \multicolumn{5}{|l|}{ Physical } \\
\hline - Temperature & Thermal & & & \\
\hline \multicolumn{5}{|l|}{ Heat } \\
\hline Sublethal & Mild & Sulfolobus islandicus & Hyperthermophylic Archaea Crenarchaeota & 34 \\
\hline Lethal & & Bacillus cereus & Bacteria Gram + & 45 \\
\hline Cold & Hypothermic/Cooling & & & \\
\hline Above $0^{\circ} \mathrm{C}$ & Chilling & Arthrobacter globiformis & Psychrotrophic Bacteria Gram + & 8 \\
\hline Under $0^{\circ} \mathrm{C}$ & & Geotrichum candidum & Fungi Yeast & 42 \\
\hline \multicolumn{5}{|l|}{ - Drying } \\
\hline Freeze drying & Lyophyliz(s)ation & Lactobacillus reuteri & Bacteria Gram + & 40 \\
\hline Spray drying & Atomiz(s)ation & Beijerinckia sp. & Bacteria Gram - & 11 \\
\hline - Pressure & Barotolerance/Pressurization & Saccharomyces cerevisiae & Fungi Yeast & 15 \\
\hline \multicolumn{5}{|c|}{ Gravitational } \\
\hline Microgravity & & Streptomyces clavuligerus & $\begin{array}{l}\text { Bacteria } \text { Gram }+ \\
\text { Actinomycete Streptomycete }\end{array}$ & 18 \\
\hline Hypergravity & & Synechocystis sp. & Cyanobacteria & 16 \\
\hline \multicolumn{5}{|l|}{ - Radiation } \\
\hline Ultraviolet & & Saccharomyces cerevisiae & Fungi Yeast & 29 \\
\hline Visible light & & Escherichia coli & Bacteria Gram - & 53 \\
\hline - Ultrasonic & Acoustic & Pseudomonas aeruginosa & Bacteria Gram - & 49 \\
\hline - Electrical stress & & Pseudomonas oleovorans & Bacteria Gram - & 2 \\
\hline - Magnetic field & & Escherichia coli & Bacteria Gram - & 38 \\
\hline \multicolumn{5}{|l|}{ - Mechanical stress } \\
\hline Vibration & Vibrational & Escherichia coli & Bacteria Gram - & 1 \\
\hline Shear & Shearing & $\begin{array}{l}\text { Lactobacillus delbrueckii } \\
\text { subsp. bulgaricus }\end{array}$ & Bacteria Gram + & 3 \\
\hline \multicolumn{5}{|l|}{ Chemical } \\
\hline - Starvation & $\begin{array}{l}\text { Deprivation/Nutritional stress/ } \\
\text { Nutrient limitation }\end{array}$ & & & \\
\hline \multicolumn{5}{|c|}{ रुपाता } \\
\hline Glucose starvation & & Lactococcus lactis & Bacteria Gram + & 30 \\
\hline Energy stress & & Macrophomina phaseolina & Fungi Mould Phytopathogen & 27 \\
\hline Oxygen & $\begin{array}{l}\text { Anaerobic stress/ Hypoxia/ } \\
\text { Anaerobiosis/Low oxygen/ } \\
\text { Anoxia }\end{array}$ & Penicillium chrysogenum & Fungi Mould & 36 \\
\hline Iron & & Helicobacter pylori & Bacteria Gram - & 37 \\
\hline \multicolumn{5}{|l|}{ - Overexposure } \\
\hline Heavy metal & & Chlamydomonas reinhardtii & Microalgae Chlorophyta & 25 \\
\hline Bile salt & & Enterococcus faecalis & Bacteria Gram + & 51 \\
\hline Ethanol & & Oenococcus oeni & Bacteria Gram + & 65 \\
\hline Organic solvent & & Pseudomonas putida & Bacteria Gram - & 57 \\
\hline Sanitizer & & Yersinia enterocolitica & Bacteria Gram - & 19 \\
\hline Food Preservatives & & Gluconobacter oxydans & Bacteria Gram - & 17 \\
\hline Nitrosating agents & Nitrosative & Giardia intestinalis & Protozoa Flagellate & 33 \\
\hline \multicolumn{5}{|l|}{ - Oxidative } \\
\hline $\mathrm{R}-\mathrm{O}-$ & Oxidation/Superoxide & Streptococcus thermophilus & Bacteria Gram + & 63 \\
\hline $\mathrm{O}_{2}$ & $\begin{array}{l}\text { Oxygen peroxide/ } \\
\text { Peroxidative/Hydroperoxide }\end{array}$ & Bifidobacterium sp. & Bacteria Gram + & 39 \\
\hline - Reductive & & Saccharomyces cerevisiae & Fungi Yeast & 66 \\
\hline - Acid & Low $\mathrm{pH}$ & & & \\
\hline Strong acid & & Rhodococcus equi & Bacteria Gram + Actinomycete Nocardioform & 7 \\
\hline Weak acid & & Zygosaccharomyces bailii & Fungi Yeast & 47 \\
\hline - Alkaline & & Enterococcus faecalis & Bacteria Gram + & 21 \\
\hline \multicolumn{5}{|l|}{ - Low water activity } \\
\hline Dessication & $\begin{array}{l}\text { Dehydration /Drought } \\
\text { tolerance/Water stress }\end{array}$ & Pseudomonas fluorescens & Bacteria Gram - & 56 \\
\hline Hyperosmolarity & Salinity/ $\mathrm{NaCl}$ & Lactobacillus plantarum & Bacteria Gram + & 48 \\
\hline - High water activity & Hypoosmotic/Dilution stress & Dunaliella tertiolecta & Microalgae Chlorophyta & 23 \\
\hline
\end{tabular}

